\title{
Medievalista
}

Online

$25 \mid 2019$

Número 25

\section{Itinerario de una lista. De la Hispania visigoda a Italia beneventana, del Liber Iudiciorum al Pseudo- Isidoro}

The route of a list. From visigothic Hispania to beneventan Italia, from Liber Iudiciorum to Pseudo Isidore

\section{William Trouvé}

\section{OpenEdition}

Journals

Edición electrónica

URL: http://journals.openedition.org/medievalista/1735

DOI: $10.4000 /$ medievalista. 1735

ISSN: 1646-740X

Editor

Instituto de Estudos Medievais - FCSH-UNL

Referencia electrónica

William Trouvé, «Itinerario de una lista. De la Hispania visigoda a Italia beneventana, del Liber

Iudiciorum al Pseudo-Isidoro », Medievalista [En línea], 25 | 2019, Puesto en línea el 17 marzo 2019 consultado el 19 abril 2019. URL : http://journals.openedition.org/medievalista/1735 ; DOI : 10.4000/ medievalista. 1735

Este documento fue generado automáticamente el 19 abril 2019.

(C) IEM 


\title{
Itinerario de una lista. De la Hispania visigoda a Italia beneventana, del Liber Iudiciorum al Pseudo-Isidoro
}

\author{
The route of a list. From visigothic Hispania to beneventan Italia, from Liber \\ Iudiciorum to Pseudo Isidore
}

William Trouvé

\section{NOTA DEL EDITOR}

Data recepção do artigo / Received for publication: 14-08-2017

Data aceitação do artigo / Accepted in revised form: 06-07-2018

1 El manuscrito 1 del monasterio de Montecassino contiene una lista de reyes visigodos elaborada durante la segunda mitad del siglo VIII ${ }^{1}$. El manuscrito fue confeccionado en una época posterior y, dado que el copista empleó una escritura beneventana, se puede afirmar que el códice fue copiado en Italia en el siglo $\mathrm{XI}^{2}$. ¿Cómo explicar la aparición de esta lista en el mundo cultural beneventano? Es evidente que la presencia de este texto de origen visigodo confirma la existencia de intercambios culturales entre Hispania e Italia gracias a intelectuales, monjes y viajeros de la Alta Edad Media. Para entender cómo la lista pudo llegar a esta zona hemos de identificar y confrontar la historia del manuscrito y la de la lista.

2 La lista regia visigoda ha estado asociada al Liber Iudiciorum, un código legislativo visigodo publicado por Recesvinto en 654 y revisado por Ervigio en $681^{3}$. Todos los ejemplares conservados de este libro y elaborados entre los siglos VIII y IX contienen esta lista: son testimonios pertenecientes a la tradición catalana u oriental del Liber ${ }^{4}$. El manuscrito italiano no es un libro del Liber Iudiciorum, sino que se trata de un ejemplar de las Falsas 
Decretales atribuidas al Pseudo-Isidoro, que tiene su origen en el mundo carolingio del siglo IX. Estas decretales son una colección de falsas cartas papales, confeccionada en una zona que abarca el monasterio de Corbie, en el norte de Francia, y las provincias del noreste de ese mismo territorio entre el 830 y el 870. Los autores pretendían defender la figura del obispo frente al poder imperial carolingio y a los arzobispos ${ }^{5}$.

¿Por qué la lista visigoda fue desplazada de un código "bárbaro" hacia una colección de derecho canónico? Es una pregunta que nos asalta, cuando leemos la lista regia. En general, los testimonios conservados de la lista se limitan a recoger los nombres de los reyes y la duración del reinado. El texto de Montecassino pertenece a una familia textual que se abre con una introducción sobre la llegada de los visigodos en Hispania; a continuación, la lista empieza con los primeros soberanos, Atanarico, Alarico, Ataúlfo, Sigerico, etc., pero no se cierra con el último rey, Rodrigo, sino con la unción de Ervigio. Además, la lista copiada en Italia aporta dos informaciones que no aparecen en ninguna otra lista; es decir, menciona los nombres de Isidoro de Sevilla, de l'emperador Mauricio y de Gregorio Magno después de los reinados de Leovigildo y de Sisebuto.

¿Podemos interpretar estas modificaciones con la llega de la lista dentro de la colección de derecho canónico? Vamos a intentar elucidar cómo fue el camino de la lista visigoda desde Hispania hasta Italia, pero también el de la colección legislativa visigoda hasta el derecho eclesiástico. Veremos que las transformaciones de la lista están plenamente vinculadas a su transmisión manuscrita e intelectual.

\section{1. ¿Cómo explicar la presencia de una lista visigoda del siglo VIII dentro de Italia del siglo XI?}

\subsection{Los rasgos comunes de algunos manuscritos pseudoisidorianos}

El códice de Montecassino es un manuscrito bastante grande, que mide $405 \mathrm{~mm}$ x 295mm. Se compone de 225 folios paginados. El texto está escrito a doble columna. Se emplean iniciales y un íncipit en rojo. La copia está cuidada con algunas miniaturas. Es un libro precioso.

6 El contenido del códice ha sido estudiado por los especialistas de las decretales pseudoisidorianas. Existen varias versiones de esta colección, según la presencia o ausencia de una de los tres partes del Pseudo-Isidoro. Desde el estudio de Paul Hinschius en 1869, el manuscrito de Montecassino pertenece a la clase A/B, es decir que el códice mantiene la primera parte (pp. 3-128) y la tercera parte (pp. 131-402) completas, así como la tabla de los índices de la segunda parte (pp. 121-131) de las Falsas Decretales ${ }^{6}$.

7 Junto a esos documentos, otros materiales han sido copiados a continuación:

1. Los Capitula Angilramni (pp. 402-408)

2. Un extracto de la Historia Tripartita de Casiodoro (V, 17: Disputatio imperatoris Constantii et Liberii romani pontificis) (p. 408-409)

3. Actas del Concilio de Calcedonia (a. 451) (p. 409-429)

4. Concilio Quinisexto (Constantinopla, a. 692) (p. 429-431)

5. Un extracto de la Historia Tripartita de Casiodoro (XII, 8, 10-11) (p. 431-432)

6. Un fragmento de una falsa decretal atribuida a Pelayo II (p. 433)

7. La lista regia visigoda intitulada Chronica regum Visigothorum (p. 433-434) 
8. Cartas atribuidas a Gregorio Magno (p. 434-440)

9. Epistola Isidori Hispalensis episcopi ad Laudofredum (p. 440-441)

10. Epistola Isidori Hispalensis episcopi ad Masonem (p. 441-443)

11. Matrimonii ratione (p. 443)

12. Etymologiae, VIII, 5-6 (p. 443-447)

13. Canones I y X de Toledo XI (p. 447-448).

Estos trece elementos abordan asuntos religiosos y teológicos, puesto que se trata de algunos extractos de concilios, de derecho canónico o de textos de los Padres de la Iglesia. La lista regia (pp. 433-434) se encuentra entre ellos. Todavía no podemos explicar por qué se presenta la lista aquí, pero el contenido del códice nos permite de hacer paralelos con otros manuscritos pseudoisidorianos más antiguos.

Uno de ellos es el París, BnF, lat. 1557 que tiene también la lista regia. Este códice fue elaborado en el último cuarto del siglo IX, posiblemente entre 872 y 882 , en la zona de Reims ${ }^{7}$. El contenido del manuscrito es casi semejante al de Montecassino:

\begin{tabular}{|c|c|}
\hline París, BnF, lat. 1557 & Montecassino, 1 \\
\hline / & Primera parte del Pseudo-Isidoro (p. 3-128) \\
\hline / & $\begin{array}{l}\text { Tabla de los índices de la segunda parte del Pseudo- } \\
\text { Isidoro (p. 129-131) }\end{array}$ \\
\hline / & Tercera parte del Pseudo-Isidoro (p. 131-402) \\
\hline / & Capitula Angilramni (p. 402-408) \\
\hline Actas de Toledo VIII (f.1-7) & / \\
\hline / & Casiod., Hist. Trip., V, 17 (p. 408-409) \\
\hline Carta de Gregorio IV (f. 7-9) & / \\
\hline $\begin{array}{l}\text { Extractos del Concilio de Calcedonia } \\
\text { (f.9r-18ra) }\end{array}$ & Extractos del Concilio de Calcedonia (p. 409-429) \\
\hline Concilio Quinisexto (f.18ra-19ra) & Concilio Quinisexto (p. 429-431) \\
\hline $\begin{array}{l}\text { Casiod., Hist. Trip., XII, 8, 10-11 (f. } \\
\text { 19r-20r) }\end{array}$ & Casiod., Hist. Trip., XII, 8, 10-11 (p. 431-432) \\
\hline Epistola Pelagii II (f. 20r-20v) & Epistola Pelagii II (p. 433) \\
\hline Lista de los reyes visigodos (f. 20v) & Lista de los reyes visigodos (p. 433-434) \\
\hline Florilegium Gregorii (f. 20v -24v) & $\begin{array}{l}\text { Florilegium Gregorii (p. 434), otras cartas } \\
\text { atribuidas a Gregorio Magno (p. 434-440) }\end{array}$ \\
\hline Concilio de Nicea (a. 670-685) (f. 24v-27v) & / \\
\hline
\end{tabular}




\begin{tabular}{|l|l|}
\hline$/$ & $\begin{array}{l}\text { Epistola Isidori Hispalensis episcopi ad Laudofredum (p. } \\
440-441)\end{array}$ \\
\hline$/$ & $\begin{array}{l}\text { Epistola Isidori Hispalensis episcopi ad Masonem (p. } \\
441-443)\end{array}$ \\
\hline De matrimonii ratione (f. 27v-28) & De matrimonii ratione (p. 443) \\
\hline Etymologiae, VIII, 5-7 (f. 28-30v) & Etymologiae, VIII, 5-6 (p. 443-447) \\
\hline Toledo XI, c. X (f. 30-31) & Toledo XI c. I y X (p. 447-448) \\
\hline $\begin{array}{l}\text { Cartas de León III, Benedicto III, Nicolás I } \\
\text { y Adriano II (f. 31r-96v) }\end{array}$ & \\
\hline
\end{tabular}

10 Ambos manuscritos comparten los textos siguientes:

1. Actas del Concilio de Calcedonia

2. Concilio Quinisexto

3. Casiod., Hist. Trip., XII, 8, 10-11

4. Epistola Pelagii II

5. Lista regia visigoda

6. Florilegium atribuido a Gregorio Magno

7. De matrimonii ratione

8. Etymologiae, VIII, 5-7

9. Toledo XI, c. X.

11 La diferencia entre ambos códices se debe a que el parisino tiene otros textos teológicos, mientras que el de Montecassino incorpora sólo material pseudoisidoriano. No obstante, si nos fijamos en los textos comunes, se observa que el orden de aparición es idéntico. Además, hay que acordar que el manuscrito de París fue elaborado junto con otro códice del siglo IX: el actual París, BnF, lat. $9629^{8}$. Ambos formaron, pues, un único volumen. Conviene señalar que el contenido de París, BnF, lat. 9629 es muy semejante al italiano de Montecassino. El códice parisino conserva el final de la primera parte del Pseudo-Isidoro y la totalidad de la tercera parte ${ }^{9}$; pero además finaliza con los Capitula Angilramni y la Disputatio Constantii Imperatoris et Liberii papae (Cassiod, Hist. Trip., 5, 17), que son los dos textos con los que se abre el manuscrito de Montecassino.

12 Hay que hacer notar que, si bien el contenido de ambos códices difiere en algún aspecto, los textos comunes permiten afirmar que los dos manuscritos pertenecen a una misma familia, lo que ya había subrayado Karl-Georg Schon en su estudio de los Capitula Angilramni ${ }^{10}$.

\subsection{Historia breve de la composición del Pseudo-Isidoro}

13 Así pues, el origen de la lista copiada en Italia se remonta al mundo carolingio del siglo IX. Es necesario contar la historia de la composición, así como de la circulación, del llamado Pseudo-Isidoro para comprender cómo la lista llegó a incluirse entre sus materiales.

14 Según la reciente interpretación de Eric Knibbs, el Pseudo-Isidoro habría sido elaborado en muchas fases ${ }^{11}$. Cada fase está vinculada a una de las tres partes que componen el 
Pseudo-Isidoro. Los historiadores aún no han conseguido a identificar toda la historia de la colección. A día de hoy, sólo pueden explicarse los primeros elementos que fueron elaborados en el monasterio de Corbie, bajo la dirección del abad Wala, en los años 830. El abad era partidario de Lotario I y estaba en contra, pues, de Luís Pío. Posteriormente, los alumnos de Wala desarrollaron y aumentaron el contenido, durante los años 850. En la misma época, un segundo centro produjo otros textos. La recopilación de los textos tuvo lugar en una fecha y en una localización todavía hoy desconocidos. A tal recopilación se la conoce hoy como Pseudo-Isidoro, cuyo primer documento se localiza por primera vez en 869 en la zona de Reims. En esta época, el Pseudo-Isidoro fue utilizado en la lucha ideológica que enfrentó al arzobispo Hincmar de Reims contra su sobrino el obispo Hincmar de Laon. Los manuscritos París lat. 9629 y lat. 1557 fueron elaborados en aquel ambiente de enfrentamiento.

¿Cómo se introduce la lista en esta lucha? ¿Tenemos que concluir que fue añadida al final de los años $860 ?$ ¿O podemos suponer que ya fue usada antes? Ciertos elementos que acompañan la lista fueron incluidos en los manuscritos pseudoisidorianos al principio de la empresa; así, sucedió con los Capitula Angilramni, los extractos del Concilio de Calcedonia, y las decretales de Gregorio, que posiblemente fueron ya tenidos en cuenta desde Corbie ${ }^{12}$.

Además, hay que señalar que las fuentes hispánicas son numerosas en la colección. Primero, las decretales se basan en la Hispana Gallica que es una forma derivada de la Collectio Hispana, difundida en Galia entre los siglos VIII y IX ${ }^{13}$. También, se encuentran extractos del concilio de Toledo XI, así como algunos textos atribuidos a Isidoro. Los autores del Pseudo-Isidoro dispusieron de materiales hispánicos: podemos imaginar que la lista procede de esos recursos y fue empleada en esta misma época.

Pero, los indicios para probar que la lista fue usada desde los años 830 y unida a los materiales visigodos son débiles. Parece más cierto que su presencia, al lado de otros textos visigodos, es más una cuestión de refuerzo para confirmar la autoridad de los demás textos hispánicos, que de recursos primarios empleados por los autores del Pseudo-Isidoro. La inclusión de la lista pudo tener lugar más tarde, en torno a los años 860 o 870.

\section{3. ¿De dónde viene la lista?}

Lo más probable es que la lista provenga de los manuscritos del Liber Iudiciorum copiados en el mundo carolingio. Conocemos dos fechados del siglo IX: el París, BnF, lat. 4668 y París, BnF, lat. 4418. Bernhard Bischoff propuso que París lat. 4668 fue confeccionado en Narbonense, en el tercer tercio de este siglo. Fue conservado en el monasterio de SaintRemi de Reims al menos desde el siglo $\mathrm{XI}^{14}$. Respecto al París lat. 4418, copiado en el segundo tercio del siglo IX ${ }^{15}$, su rico contenido planteó más problemas. Se trata de un código de leges barbarorum que mantiene una versión de la Lex Salica, Lex Burgundionum, Lex Alamanorum, del Epitome Aegidii, así como del Epitome Iuliana y, por supuesto, del Liber Iudiciorum. Este libro se presenta como un libro de lujo, tanto por su tamaño como la elegancia de su escritura. Durante mucho tiempo, algunos historiadores supusieron que el manuscrito procedía de un scriptorium oficial, próximo a la corte ${ }^{16}$, pero la hipótesis ha sido rechazada por Karl Ubl recientemente ${ }^{17}$. Y. García López recordó que este tipo de códigos legales se encontraban en el sur de Francia; pero reconoce que es difícil saber si el 
manuscrito fue elaborado en el norte de Francia y una copia habría llegado al sur o si, al contrario, un códice del sur llegó hasta el norte ${ }^{18}$.

A falta de informaciones más detalladas sobre el origen de cada uno de los códices, no existen pruebas concretas que permitan afirmar que el Liber Iudiciorum fuera conocido en Francia. No obstante, la emigración de intelectuales visigodos después de la conquista musulmana permite suponer que algunos ejemplares puedan haber sido transmitidos a Francía ${ }^{19}$. En este sentido apunta también la inclusión de una lista de reyes visigodos en el Pseudo-Isidoro, recopilación de época carolingia. En resumen, a la vista de lo expuesto, se puede afirmar que es posible que los autores del Pseudo-Isidoro hubieran empleado un ejemplar del código legal visigodo que circuló por el territorio carolingio para extraer de él la lista. Tal vez, fue en la región de Reims donde se realizó una copia del Pseudo-Isidoro conteniendo la lista.

\subsection{Conclusiones sobre la transmisión de la lista}

Desde el sur de Francia, unos ejemplares del Liber Iudiciorium y de la lista regía llegaron al noreste de Francia. En esta región, los copistas del Pseudo-Isidoro tomaron de un código legal visigodo la lista que más tarde añadirían a un ejemplar pseudoisidoriano que contenía ya otros textos de origen hispánico. El motivo de la inclusión de la lista era apoyar y reforzar la autoridad de los documentos visigodos. El primer testimonio del conjunto se encuentra en un único volumen, elaborado entre el 872 y el 882, en la zona de Reims, es decir los actuales París, BnF, lat. 1557 y 9629. Un ejemplar semejante viajó hasta Italia en fecha desconocida. De él se hizo una copia en la zona de Benevento durante el siglo XI, cuando el papado y el episcopado empiezan a imponer su autoridad, anunciando el apogeo de las copias del Pseudo-Isidoro en el siglo XII, antes de la realización del Decreto de Graciano ${ }^{20}$.

\section{2. ¿Cómo explicar las presencias de Isidoro, de Mauricio y de Gregorio en la lista?}

21 Como ya hemos explicado en la introducción, la lista regia del manuscrito de Montecassino muestra los nombres del papa Gregorio Magno, del emperador Mauricio y del obispo Isidoro de Sevilla. Si comparamos esta lista con los testimonios carolingios del siglo IX, nos damos cuenta de que:

1) las listas incluidas con el Liber Iudiciorum (París lat. 4668 y 4418) pertenecen a la misma rama textual que la lista italiana. Tienen una introducción, un anuncio y finalizan con la unción de Ervigio. También contienen otras informaciones, como la vacante del poder (c.24) o la muerte de Cindasvinto (c.40 ${ }^{21}$. Lo que también se encuentra en el París lat. 1557.

23 2) Los nombres de Gregorio, Mauricio e Isidoro no aparecen en las listas del Liber Iudiciorum. En cambio, París lat. 1557 menciona también esos tres nombres, lo que permite suponer que esas figuras fueron añadidas cuando la lista se asoció al Pseudo-Isidoro. Las modificaciones aparecen así en el texto:

c.26: Liuuigildus regnauit annos XVIII. Iste Leuuigildus temporibus Gregorii papae

et Mauricii imperatoris fuit. 
c.32: Sissebodus regnauit annos VIII menses XI dies XVI. Sisebudi temporibus fuit Isidorus episcopus.

24 Vamos a analizar el papel de esas inclusiones en el texto y así identificar los motivos del copista para incluir esas modificaciones. Luego, intentaré explicar por qué la inclusión solo afectó Isidoro, Gregorio Magno y Mauricio.

\subsection{Un uso práctico de la lista: ¿sincronizar algunas figuras históricas?}

Las modificaciones de un texto pueden ser el resultado de factores diferentes. Puede tratarse de corregir el texto para hacerlo más claro, amplificarlo para actualizar el texto $\mathrm{u}$ omitir elementos en el caso de una damnatio memoriae, por ejemplo. El caso de la lista asociada al Pseudo-Isidoro no entra en ninguno de estos supuestos. Tenemos que analizar las frases añadidas.

Cada frase corresponde a una oración independiente. Se coloca después de la oración que trata del rey. El hecho de que sólo se encuentren dos oraciones así demuestra que son añadidas. Ambas oraciones están construidas diferentamente. Repiten el nombre del rey que está seguido por el término temporibus en ablativo, que tiene un valor temporal. A continuación, se añaden los nombres de los personajes con el verbe esse en perfecto. Podemos concluir que son frases cortas que sincronizan la vida de varios personajes. Pero, en la primera oración, el nombre del rey se encuentra en nominativo mientras que en la segunda está en genitivo. Hay una inversión del sujeto lo cual podría significar que estas dos adiciones proceden de otros tantos autores distintos. En cualquier caso, está claro que los visigodos sirven de hito cronológico.

El texto no es siempre correcto. Es cierto que Sisebuto (612-621) reinó en parte durante el obispado de Isidoro de Sevilla (599-636) y que Leovigildo (572-586) terminó su reinado al principio de la llegada al trono del emperador Mauricio (582-602). Sin embargo, no es correcto afirmar que Leovigildo reinó durante el mandato del papa Gregorio Magno (590-604): Leovigildo ya había muerto cuando el papa accedió al solio. Sin embargo, Mauricio y Gregorio gobernaron en la misma época. Eso puede explicar el error del copista. Posiblemente, sabía que Mauricio había reinado al mismo tiempo que Leovigildo y Gregorio Magno. Eligió vincular al rey visigodo con el papa romano. Parece evidente que el autor de esta lista se equivocó al intentar conectar a esos tres personajes; no se trata, pues, de una manipulación cronológica ${ }^{22}$. El propósito del copista carolingio fue claramente práctico y cronológico, más que una preocupación historiográfica. Intentó identificar la cronología de esos tres personajes como ayuda al lector, posiblemente para fechar sus textos.

\subsection{Una lista adaptada al contenido del manuscrito}

¿Por qué el autor de esas líneas eligió introducir a Gregorio, Mauricio e Isidoro y no otras figuras antiguas? Su actuación no puede ser fortuita. Siempre hemos de tener en cuenta que las modificaciones están vinculadas al nuevo ambiente codicológico.

La lista se encuentra junto a textos isidorianos. Ambos manuscritos mantienen extractos del libro VIII de las Etimologías, en concreto de los capítulos 5 y 6 que tratan de las herejías cristianas y de los filósofos griegos. Además, el códice de Montecassino tiene dos epístolas atribuidas a Isidoro que están dirigidas a Leudefredus y Massona. El nombre de 
Gregorio aparece en un Florilegium que sigue directamente a continuación de la lista regia en los dos manuscritos; la autoría de tal florilegio se atribuye a Gregorio Magno. Se sabe que una de las cartas está dirigida a precisamente a Mauricio ${ }^{23}$.

Creo que los nombres de Isidoro, Gregorio y Mauricio fueron añadidos para fechar sus textos. En este caso, ¿por qué el autor no añadió los nombres del papa Pelayo II o de Casiodoro, cuyos textos aparecen también antes de la lista regía? A falta de más elementos de juicio resulta imposible responder a esta cuestión, salvo que sencillamente el copista careciera de información.

\section{A modo de conclusión}

La lista de los reyes visigodos fue elaborada en la segunda mitad del siglo VII, posiblemente en Toledo, y asociada al Liber Iudiciorum. La emigración hispánica del siglo VIII hacia el territorio carolingio llevó consigo algunos ejemplares del código legislativo. De la Septimania y la Narbonensia, los libros se difundieron hasta el noreste del reino franco. La lista fue utilizada por copistas del Pseudo-Isidoro que la emplearon como anexo a las falsas Decretales pseudoisidorianas. La introducción de la lista tenía como finalidad reforzar la autoridad de otros textos que también estaba usados como anexos. Esta operación fue realizada posiblemente al final de los años 860, en la zona de Reims.

La introducción de la lista dentro de un nuevo ambiente codicológico supuso algunas modificaciones: la introducción de las menciones al papa Gregorio Magno, así como al emperador Mauricio en relación al reinado de Leovigildo, y la de Isidoro en relación al reinado de Sisebuto. Posiblemente, el copista intentó fechar los textos de Gregorio e Isidoro sirviéndose de la lista para revalorizar la autoridad antigua de esos documentos. Con la difusión del Pseudo-Isidoro, la lista cruzó las Alpes en una fecha desconocida. En el siglo XI, en la zona cultural de Benevento, un nuevo ejemplar fue copiado, sobre el modelo de los años 860-870.

\section{BIBLIOGRAFÍA}

Fontes manuscritas

Montecassino, Archivio e Biblioteca dell'Abbazia, 1.

París, Bibliothèque nationale de France, lat. 1557.

París, Bibliothèque nationale de France, lat. 4668.

París, Bibliothèque nationale de France, lat. 4418.

Fontes impressas

Angilramnus - Capitula. Ed. Karl-Georg Schon. Hannover: Hahnsche Buchhandlung, 2006.

Laterculus regum Visigothorum. Ed. Theodor Mommsen. Monumenta Germaniae Historica: Auctores

Antiquissimi. T. XIII, Chronica Minora, vol. 3, München: MGH, 1981, pp. 464-469. 
Monachorum Ordinis S. Benedicti Abbatiae Montis Casini - Bibliotheca Casinensis seu codicum manuscriptorum qui in tabaulario Casinensi asservantur: series per paginas singillatim enucleata notis, characterum speciminibus ad unguem exemplatis aucta. Vol. I. Montecassino: ex typographia Casinensi, 1873.

Pseudo-Isidoro - Decretales. Ed. Paul Hinschius. Leipzig: Tauchnitz, 1873.

Estudos

BAUTISTA, Francisco - "Breve historiografía: listas regias y anales en la Península Ibérica (siglos VII-XII)". Talia Dixit 4 (2009), pp. 113-190.

BISCHOFF, Bernhard; EBERSOERGER, Birgit - Katalog der festländischen Handschriften des neunten Jahrhunderts (mit Ausnahme der wisigotischen). Vol. 3. Padua- Zwickau. Wiesbaden: Harrassowitzol, 2014.

CONTRENI, John J. - "Codices Pseudo-Isidoriani: The Provenance and Date of Paris, B.N. MS lat. 9629”. Viator 13 (1982), pp. 1-14.

DÍAZ Y DÍAZ, Manuel C. - "La Lex Visigothorum y sus manuscritos. Un ensayo de reinterpretación”. Anuario de historia del derecho español 46 (1976), pp. 163-224.

FUHRMANN, Horst; JASPER, Detlev - Papal letters in the early Middle Ages. Washington DC: The Catholic University of American Press, 2001.

GARCÍA LÓPEZ, Yolanda - Estudios críticos y literarios de la "Lex Wisigothorum". Alcalá de Henares: Universidad de Alcalá de Henares, 1996.

HARDER, Clara - "Der Papst als Mittel zum Zweck? Zur Bedeutung des römischen Bischofs bei Pseudoisidor". in UBL, Karl; ZIEMANN, Daniel (Ed.) - Fälschung als Mittel der Politik? Pseudoisidor im Licht der neuen Forschung. Wiesbaden: Harrassowitz, 2015.

HINSCHIUS, Paul - Decretales Pseudo-Isidorianae et Capitula Angilramni. Leipzig: Tauchnitz, 1873. INGUANEZ, Mauro - Codicum casinensium manuscriptorum catalogus. Vol. I, pars I. Montecassino: s.l., 1915.

KNIBBS, Eric - "The interpolated Hispania and the origins of Pseudo-Isidore". Zeitschrift der Savigny-Stiftung für Rechtsgeschichte: Kanonistische Abteilung 130 (2013), pp. 1-71.

MCKITTERICK, Rosamond - "Some Carolingian law-books and their functions". in MCKITTERICK, Rosamond (Ed.) - Books, scribes and learning in the Frankish kingdoms 6th - 9th centuries. Aldershot: Variorum, 1994, pp. 13-27.

SCHON, Karl-Georg - Die Capitula Angilramni: eine prozessrechtliche Fälschung Pseudoisidors. Hannover: Hahnsche Buchhandlung, 2006.

UBL, Karl - “Gab es das Leges-Skriptorium Ludwigs des Frommen?”. Deutsches Archiv für Erforschung des Mittelalters 70 (2014), pp. 43-65.

WILLIAMS, Schafer - Codices Pseudo-Isidoriani: a palaeographico-historical study. New-York: Fordham University Press, 1971.

\section{NOTAS}

1. Querría agradecer a la Pr. Eva Castro Caridad por su revisión del texto. Todas las faltas de ortografía, de gramatica son culpa mía. 
2. Monachorum Ordinis S. Benedicti Abbatiae Montis Casini - Bibliotheca Casinensis seu codicum manuscriptorum qui in tabaulario Casinensi asservantur: series per paginas singillatim enucleata notis, characterum speciminibus ad unguem exemplatis aucta. Vol. I: Montecassino: ex typographia Casinensi, 1873, pp. 1-48: lista figura en la pagína 44; INGUANEZ, Mauro - Codicum casinensium manuscriptorum catalogus. Vol. I, pars I. Montecassino: s.l., 1915, pp. 1-3; WILLIAMS, Schafer Codices Pseudo-Isidoriani: a palaeographico-historical study. New-York: Fordham University Press, 1971, p. 35, n³3.

3. Vide Laterculus regum Visigothorum. Ed. Theodor Mommsen. Monumenta Germaniae Historica: Auctores Antiquissimi. T. XIII, Chronica Minora, vol. 3, München: MGH, 1981, p. 461; BAUTISTA, Francisco - "Breve historiografía: listas regias y anales en la Península Ibérica (siglos VII-XII)”. Talia Dixit 4 (2009), pp. 119-124.

4. Son París, BnF, lat. 1557; París, BnF, lat. 4418; París, BnF, lat. 4667; París, BnF, lat. 4668; Vaticano, BAV, reg. lat. 1024. El estudio más reciente y completo sobre el Liber Iudiciorum es el de GARCÍA LÓPEZ Yolanda - Estudios críticos y literarios de la "Lex Wisigothorum". Alcalá de Henares: Universidad de Alcalá de Henares, 1996, p. 35.

5. Vide FUHRMANN, Horst; JASPER, Detlev - Papal letters in the early Middle Ages. Washington: The Catholic University of American Press, 2001, pp. 137-193; KNIBBS, Eric - "The interpolated Hispana and the origins of Pseudo-Isidore". Zeitschrift der Savigny-Stiftung für Rechtsgeschichte: Kanonistische Abteilung 130 (2013), pp. 59-64.

6. HINSCHIUS, Paul - Decretales Pseudo-Isidorianae et Capitula Angilramni. Leipzig: Tauchnitz, 1873, pp. LXV-LXII.

7. WILLIAMS, Schafer - Codices Pseudo-Isidoriani, pp. 45-46, n46; CONTRENI, John J. - "Codices Pseudo-Isidoriani: The Provenance and Date of Paris, B.N. MS lat. 9629”. Viator 13 (1982), pp. 13-14; BISCHOFF, Bernhard; EBERSOERGER, Birgit - Katalog der festländischen Handschriften des neunten Jahrhunderts (mit Ausnahme der wisigotischen). Vol. 3. Padua- Zwickau. Wiesbaden: Harrassowitzol, 2014, p. 35, p. 57, $\mathrm{n}^{\circ} 4614$.

8. CONTRENI, John J. - “Codices Pseudo-Isidoriani: The Provenance”, pp. 13-14.

9. HINSCHIUS, Paul - Decretales Pseudo-Isidorianae, p. XX; WILLIAMS, Schafer - Codices PseudoIsidoriani, pp. 45-46. La tercera parte pertenece a la clase A1 pero fecha del siglo X o XI, según Williams.

10. SCHON, Karl-Georg - Die Capitula Angilramni: eine prozessrechtliche Fälschung Pseudoisidors. Hannover: Hahnsche Buchhandlung, 2006, pp. 23-24.

11. KNIBBS, Eric - "The interpolated Hispana", p. 10.

12. HARDER, Clara - "Der Papst als Mittel zum Zweck? Zur Bedeutung des römischen Bischofs bei Pseudoisidor”. in UBL, Karl; ZIEMANN, Daniel (Ed.) - Fälschung als Mittel der Politik? Pseudoisidor im Licht der neuen Forschung. Wiesbaden: Harrassowitz, 2015, p. 182, 184.

13. KNIBBS, Eric - "The interpolated Hispana”, pp. 16-18.

14. BISCHOFF, Bernhard; EBERSOERGER, Birgit - Katalog der festländischen Handschriften, p. 100, no 4325 .

15. BISCHOFF, Bernhard; EBERSOERGER, Birgit - Katalog der festländischen Handschriften, p. 98, $\mathrm{n}^{\circ}$ 4317.

16. MCKITTERICK, Rosamond - "Some Carolingian law-books and their functions". in MCKITTERICK, Rosamond (Ed.) - Books, scribes and learning in the Frankish kingdoms 6th - 9th centuries. Aldershot: Variorum, 1994, p. 22.

17. UBL, Karl - “Gab es das Leges-Skriptorium Ludwigs des Frommen?". Deutsches Archiv für Erforschung des Mittelalters 70 (2014), p. 53-54.

18. GARCÍA LÓPEZ, Yolanda - Estudios críticos, pp. 43-44. 
19. Lo que ya dijo DÍAZ Y DÍAZ, Manuel C. - "La Lex Visigothorum y sus manuscritos. Un ensayo de reinterpretación”. Anuario de historia del derecho español 46 (1976), p. 17; GARCÍA LÓPEZ, Yolanda Estudios críticos, p. 47.

20. WILLIAMS, Schafer - Codices Pseudo-Isidoriani, p. 128.

21. La fecha de fallecimiento de Chindasvinto no aparece en París, BnF, lat. 4667, ni en los manuscritos más tardíos, tales como Escorial, Biblioteca del monasterio, M.III.2 y Z.II.2; Madrid, BnE, Vitr. 14.5; y Madrid, BRAH, 36, que también proponen continuaciones distintas de la lista. La fecha de fallecimiento de Chindasvinto es considerada la marca de un sub-grupo distinto dentro de la tradición manuscrita de la lista de los reyes visigodos.

22. La hipótesis ante la que nos encontramos, que antiguas notas marginales hayan sido incluídas en el texto por un copista, es posible pero no resuelve el problema: ¿Por qué alguien eligió sincronizar dos reinados visigoticos colocando en un mismo manuscrito textos procedentes de dos autoridades intelectuales distintas? Existe claramente un acto intencional, permitido por el cambio documental, es decir, el paso de un código legal y oficial hispánico del siglo VII, el Liber Iudiciorum, a una recopilación de falsas decretales, elaborada en territorio carolingio durante el reinado de Luis el Piadoso. En suma, la cuestión no es tanto el "cómo" sino el "por qué" estas notas fueron añadidas.

23. FUHRMANN, Horst; JASPER, Detlev - Papal letters, pp. 74-76.

\section{RESÚMENES}

Este trabajo trata sobre una copia de la lista de los reyes visigodos, localizada en dos manuscritos que contienen los Decretales del Pseudo-Isidoro: Montecassino, ms. 1 y París, BnF, lat. 1557. La asociación entre lista regia visigoda y derecho canónico carolingio resulta sorpredente, pues aquella fue creada junto al Liber Iudiciorum, código legal visigodo, publicado en el 654. Además, el texto de ambos códices presenta modificaciones frente a las otras copias de la lista, añadiendo de este modo los nombres de Gregorio Magno, del emperador Mauricio y del obispo Isidoro de Sevilla. Proponemos una identificación de la difusión manuscrita de las listas pertenecientes al Pseudo-Isidoro, así como una interpretación de las modificaciones, que revelan una intención deliberada.

This article deals with a copy of a visigothic regnal list, which is found in two manuscripts of Pseudo-Isidore: Montecassino, ms. 1 and Paris, BnF, lat. 1557. The visigothic regnal list/canon law association is surprising because the list was created with the Liber Iudiciorum, a visigothic legal code, published in 654. Moreover, the list from both these codices has modifications: the names of Gregory the Great, the emperor Maurice and the bishop Isidore of Seville were added. We propose to identify the manuscript difusion of the list, in relation to the Liber Iudiciorum and the Pseudo-Isidore, as well as to interpret these deliberate modifications.

\section{ÍNDICE}

Keywords: regnal list; Liber Iudiciorum; Pseudo-Isidore

Palabras claves: lista regia; Liber Iudiciorum; Pseudo-Isidoro 


\section{AUTOR}

\section{WILLIAM TROUVÉ}

Facultad de Filologia, Universidad de Santiago de Compostela, 15782 Santiago de Compostela, España

CERHIO, Université d'Angers, 49000 Angers, France

w-trouve@orange.fr

https://orcid.org/0000-0002-9793-1287 